\title{
JOINT CONTINUITY OF SEPARATELY CONTINUOUS FUNCTIONS
}

\author{
JENS PETER REUS CHRISTENSEN ${ }^{1}$
}

\begin{abstract}
It is shown that a separately continuous function $f: X \times Y \rightarrow Z$ from the product of a certain type of Hausdorff space $X$ and a compact Hausdorff space $Y$ into a metrizable space $Z$ is jointly continuous on a set of the type $A \times Y$, where $A$ is a dense $G_{\delta}$ set in $X$. The class of Hausdorff spaces $X$ in question is defined by a gametheoretic condition. The result improves (and simplifies the proof of) a recent result of Namioka. Many "deep" theorems in functional analysis and automatic continuity theory are easy corollaries.
\end{abstract}

0. Introduction. Let $f: X \times Y \rightarrow Z$ be a separately continuous function on the product of the Hausdorff spaces $X, Y$ into the Hausdorff space $Z$.

Under suitable conditions on the spaces involved $f$ will be jointly continuous on a "fat" subset of $X \times Y$.

The present paper is inspired by a most remarkable result of this type due to Namioka (see [6]). In his paper $Z$ is metrizable, $Y$ is compact and $X$ is regular and strongly countably complete (see below). Many theorems in automatic continuity theory and functional analysis are immediate or easy corollaries of 'Namioka's theorem'.

Under the above conditions there exists a dense $G_{\delta}$ subset $A$ of $X$ such that $f$ is jointly continuous on $A \times Y$.

We shall give a hopefully technically easier proof of this theorem for a much larger class of spaces $X$ which is closed under arbitrary products and defined by a gametheoretic condition.

1. Preliminaries. Let $X$ be a Hausdorff space. Let us consider the following "game" on $X$ by the players $\alpha$ and $\beta$. The player $\beta$ starts by choosing an open nonempty subset $U_{1}$ of $X$. Then the player $\alpha$ chooses an open subset $V_{1}$ of $U_{1}$ and a point $x_{1} \in V_{1}$ (his "move" is the pair $\left(V_{1}, x_{1}\right)$ with $\left.x_{1} \in V_{1}\right)$. Then $\beta$ chooses an open subset $U_{2}$ of $V_{1}$ (he may choose as he wishes but is expected to try to escape $x_{1}$ ). Next $\alpha$ chooses an open subset $V_{2}$ of $U_{2}$ and $x_{2} \in V_{2} \ldots$ (all the sets are required to be nonempty).

We shall fix the rule that $\alpha$ wins if any subsequence $x_{n_{p}}$ of the sequence $x_{n}$ accumulates to at least one point of the set $I=\bigcap_{n=1}^{\infty} V_{n}=\bigcap_{n=1}^{\infty} U_{n}$. Note that we permit other accumulation points outside $I$ and that the point of $I$ to which a subsequence accumulates may depend effectively on that subsequence.

Received by the editors May 13, 1980 and, in revised form, May 23, 1980 and September 19, 1980. AMS (MOS) subject classifications (1970). Primary 54C05; Secondary 54C35.

Key words and phrases. Separate and joint continuity, automatic continuity, denting points.

${ }^{1}$ Research supported by the Danish National Science Foundation. 
Of course this game is closely related to the games considered by Choquet in [2]. We shall consider only strategies for $\alpha$ depending on the previous move by $\beta$. A strategy for $\alpha$ is therefore a mapping $s: \mathcal{V} \rightarrow \mathcal{V}^{*}$ of the set of nonempty open sets $\mathcal{V}$ into the set $\mathcal{V}^{*}$ of pairs $(U, x)$ with $x \in U \in \mathcal{V}$ such that for $s(U)=$ $\left(s_{1}(U), s_{2}(U)\right)$ we have $s_{1}(U) \subseteq U$ for all $U \in \mathcal{V}$. We shall say that $s$ is a winning strategy if $\alpha$ is sure to win irrespective of the behaviour of $\beta$ provided $\alpha$ moves according to $s$.

We shall call $X$ a $\sigma$-well $\alpha$-favorable space if a winning strategy exists for $\alpha$.

We shall call $X$ a $\tau$-well $\alpha$-favorable space if a winning strategy exists in the closely related game, where $\alpha$ wins if (by definition of the rules) any subnet $x_{n}$ $(d \in D)$ of the sequence $x_{n}$ accumulates to at least one point in $I$ (defined as above).

As explanation for the above conventions we should perhaps inform the reader that the class of $\sigma$-well $\alpha$-favorable spaces is ideally suited for our proof below of the "Namioka" theorem. It does, however, not seem that this class is closed under topological products, but it is easy to show that the more restricted class of $\tau$-well $\alpha$-favorable spaces is closed under arbitrary topological products.

It should be obvious that both classes of spaces defined above are $\alpha$-favorable in the sense of Choquet (see [2]). There does not seem to be any direct relation to the class of strongly $\alpha$-favorable spaces considered by Choquet.

As in Choquet we see that a $\sigma$-well $\alpha$-favorable space is a Baire space (nonempty open sets are second category). Also complete metric spaces and locally compact Hausdorff spaces are $\tau$-well $\alpha$-favorable (the proof is almost identical with the proof of the corresponding results in Choquet [2]).

We note that the class of $\tau$-well $\alpha$-favorable spaces is closed under arbitrary products. Since the proof is very similar to the proof of the corresponding fact for $\alpha$-favorable spaces (see Choquet [2]) we shall only sketch the argument.

Let $X_{i}(i \in I)$ be a family of $\tau$-well $\alpha$-favorable spaces. In $X=\Pi_{i} X_{i}(i \in I)$ we choose arbitrarily a fixed point $x=\left(x_{i}\right)(i \in I)$. A winning strategy for $\alpha$ is defined as follows:

For a nonempty open set $U \subseteq X$ we find a cylindrical set

$$
\tilde{U}=\prod_{i} \tilde{U}_{i}
$$

(all but finitely many of the factors $\tilde{U}_{i}$ equals $X_{i}$ ) contained in $U$. If $\tilde{U}_{i}=X_{i}$ we put $h\left(\tilde{U}_{i}\right)=\left(X_{i}, x_{i}\right)$ and if $\tilde{U}_{i} \neq X_{i}$ we put $h\left(\tilde{U}_{i}\right)=s_{i}\left(\tilde{U}_{i}\right)$ where $s_{i}$ is a winning strategy in $X_{i}$. Now the first coordinate of $s(U)$ is the product of the first coordinates of the $h\left(\tilde{U}_{i}\right)$ 's and similarly for the second coordinate. That $s$ is winning is proved as in Choquet [2]. We need at this point the assumption of $\tau$-well $\alpha$-favorable space. We just take a universal subnet of the sequence of points in $X$ and apply the assumption coordinatewise. This would not work for sequences!

We shall now discuss the class of spaces considered by Namioka (see [6]) and we shall see that the class of $\sigma$-well $\alpha$-favorable spaces does indeed contain the "strongly" countably complete regular Hausdorff spaces "considered" by Namioka. 
A Hausdorff space $X$ is called "strongly countably complete and regular" if it is regular in the usual sense and there exists a sequence $\mathbb{Q}_{n}$ of open coverings of $X$ (it will be convenient, as we shall do, to let $\mathbb{Q}_{0}=\{X\}$ ) with the property that for any sequence of nonempty closed sets

$$
F_{0} \supseteq F_{1} \supseteq F_{2} \supseteq \ldots
$$

with $F_{n} \subseteq U_{n} \in \mathbb{Q}_{n}$ for each $n$, we have $\bigcap_{n=1}^{\infty} F_{n} \neq \varnothing$.

Suppose we make the stronger assumption that for any net $x_{d} \in F_{n_{d}}$ with $n_{d} \rightarrow \infty$, we have that the net $x_{d}(d \in D)$ has an accumulation point. We then get a more restricted class of spaces which we call "strongly $\tau$-complete and regular". It is not difficult to show that for completely regular spaces the last concept coincides with the so-called Cech-complete spaces.

Suppose now that $X$ is a "strongly countably complete and regular" Hausdorff space. We shall prove that $X$ is $\sigma$-well $\alpha$-favorable. Therefore our theorem below does contain Namioka's theorem and is of course true also for an arbitrary product of $\tau$-well $\alpha$-favorable spaces. We define the strategy $s$ for the player $\alpha$ as follows. If $A$ is a subset of $X$ let $n(A)=\sup \left\{k\right.$ : for each $n<k, \exists U_{n} \in \mathbb{Q}_{n}$ with $\left.A \subseteq U_{n}\right\}$.

Let $U$ be an open nonempty set in $X$. We let the first coordinate of $s(U)$ be any open nonempty subset $V$ of $U$ with $\bar{V} \subseteq U$ and $n(\bar{V})>n(\bar{U})+1$. We can choose any point in $V$ as second coordinate of $s(U)$. It is indeed easy to show that $s$ is a winning strategy for the $\sigma$-game.

\section{Proof of the main theorem.}

THEOREM 1. Let $f: X \times Y \rightarrow Z$ be a separately continuous function on the product of the $\sigma$-well $\alpha$-favorable space $X$ and the compact Hausdorff space $Y$ taking values in the metric space $(Z, d)$. Then there exists a dense $G_{\delta}$ subset $A$ of $X$ such that $f$ is jointly continuous at any point of $A \times Y$.

Proof. The proof proceeds in a few steps.

Step 1. Reduction to the case where $Z$ equals the interval $[-1,1]$ with its usual metric.

Let us consider the compact Hausdorff space

$$
K=\left\{k: Z \rightarrow[-1,1]\left|\forall_{z_{1}, z_{2} \in Z}:\right| k\left(z_{1}\right)-k\left(z_{2}\right) \mid<d\left(z_{1}, z_{2}\right)\right\} .
$$

Of course $K$ is compact in the topology of pointwise convergence. On $X \times(Y \times$ $K)$ we define the function $g$ by $g(x,(y, k))=k(f(x, y))$.

If $f: X \times Y \rightarrow Z$ fulfills the conditions of the theorem it is easily seen that $g$ is separately continuous on the product of $X$ and the compact Hausdorff space $Y \times K$. Also it is seen that the conclusion for $g$ in the theorem would yield the same for $f$. In order to convince the reader about this point let $z_{\delta}(\delta \in \Delta)$ be a net in $Z$ such that the limit equation $\lim _{\delta} k\left(z_{\delta}\right)=k\left(z_{0}\right)$ holds for all $k \in K$. If we put $k(z)=d\left(z, z_{0}\right) \wedge 1$ it becomes obvious that $z_{\delta} \rightarrow z_{0}$. From this our assertion follows easily. 
Step 2. Proof of the main lemma:

MAIN LEMMA. Let $f_{n} \in C(Y)$ be any uniformly bounded sequence of continuous real valued functions on the compact Hausdorff space $Y$. Suppose that any subsequence $f_{n_{p}}$ has at least one continuous function as an accumulation point in the topology of pointwise convergence for functions on $Y$. Then if $h$ is a continuous function which is an accumulation point of $f_{n}$ in the pointwise topology, we can choose a subsequence $f_{n_{p}}$ of $f_{n}$ which tends pointwise to $h$ and hence converges weakly to $h$.

Proof. On $Y$ we define the equivalence relation

$$
y_{1} \sim y_{2} \Leftrightarrow \forall_{n} f_{n}\left(y_{1}\right)=f_{n}\left(y_{2}\right) .
$$

The set $\tilde{Y}$ of equivalence classes is a compact metric space in an obvious way and each $f_{n}$, as well as each continuous accumulation point of $f_{n}$, can be naturally identified with a continuous function on $\tilde{Y}$ (which we denote by $\tilde{f}_{n}$ ). Also $h$ induces a continuous function on $\tilde{Y}$ denoted by $\tilde{h}$. Let $\tilde{y}_{k} \in \tilde{Y}$ be a sequence which is dense in $\tilde{Y}$. We choose a subsequence $f_{n_{p}}$ such that $\tilde{f}_{n_{p}}\left(\tilde{y}_{k}\right) \rightarrow \tilde{h}\left(\tilde{y}_{k}\right)$ for each $k$. Let us convince ourselves that already $f_{n_{p}}$ tends to $h$ pointwise on $Y$. Suppose this were not true for $y_{0} \in Y$. We could then choose a subsequence $f_{n_{q}}$ of $f_{n_{p}}$ such that the limit of $f_{n_{q}}\left(y_{0}\right)$ is different from $h\left(y_{0}\right)$. The subsequence $f_{n_{q}}$ has a continuous accumulation point $h_{1}$ which induces a continuous function $\tilde{h}_{1}$ on $\tilde{Y}$. But the functions $\tilde{h}$ and $\tilde{h}_{1}$ coincide on a dense subset of $\tilde{Y}$; hence they are equal. Consequently, $h_{1}=\boldsymbol{h}$, which contradicts $h\left(y_{0}\right) \neq \lim f_{n_{q}}\left(y_{0}\right)=h_{1}\left(y_{0}\right)$.

It is not difficult to show that $f_{n}$ is weakly conditionally compact under the conditions of the lemma. We shall not need this but only the fact that if $f_{n} \rightarrow h$ pointwise then $f_{n} \rightarrow h$ weakly (use the boundedness, the Lebesgue theorem on dominated convergence and Riesz representation theorem). Consequently suitable convex combinations of the $f_{n}$ 's tend uniformly to $h$.

Step 3. Suppose now that $f$ is separately continuous on the product $X \times Y$ of the $\sigma$-well $\alpha$-favorable space $X$ and the compact Hausdorff space $Y$ and takes values in $[-1,1]$. We consider the associated mapping

$$
F: X \rightarrow C(Y)
$$

defined by $F(x)(y)=f(x, y)$.

Let the local oscillation of $F$ at $x \in X$ be defined by

$$
\alpha_{F}(x)=\inf \left\{\sup \left\{\left\|F\left(x_{1}\right)-F\left(x_{2}\right)\right\|_{\infty} \mid x_{1}, x_{2} \in U \in \mathcal{Q}(x)\right\}\right\}
$$

where the infinum is taken over all neighborhoods of $x$. Since $\alpha_{F}$ is obviously upper semicontinuous we see that $A=\left\{x \in X \mid \alpha_{F}(x)=0\right\}$ is a $G_{\delta}$ set. It is easy to see that $A$ is exactly the set of $x \in X$ such that $x \times Y$ consists entirely of joint continuity points of $f$.

The space $X$ is Baire. Therefore, if $A$ is not dense in $X$, then there exists $k>0$ such that $\left\{x: \alpha_{F}(x)<k\right\}$ is not dense, i.e., $\alpha_{F}>k>0$ on a nonempty open set $U$. Replacing $U$, which is $\sigma$-well $\alpha$-favorable, with $X$, we have reduced the proof to the derivation of a contradiction from the assumption that $\alpha_{F}(x)>k>0$ for all $x \in X$. 
Let us start the topological "game" between the players $\alpha$ and $\beta$. Naturally $\alpha$ chooses to move according to some winning strategy $s$. The player $\beta$ chooses the following strategy:

In the first move $\beta$ chooses his set $U_{1}$ to be the whole space $X$. Assume that the moves $U_{1}, s\left(U_{1}\right)=\left(V_{1}, x_{1}\right), \ldots, U_{n-1}, s\left(U_{n-1}\right)=\left(V_{n-1}, x_{n-1}\right)$ have been made with $n \geqslant 2$. In the move number $n$ for $\beta$, the compact set

$$
H_{n}=\operatorname{conv}\left(\left\{F\left(x_{1}\right), F\left(x_{2}\right), \ldots, F\left(x_{n-1}\right)\right\}\right)
$$

is considered. Let us show that

$$
C_{n}=\left\{x \in X \mid \delta\left(F(x), H_{n}\right)<k / 3\right\}
$$

has empty interior in $X$ (of course this set is closed). To see this we remark that

$$
\tilde{H}_{n}=\left\{f \in C(Y) \mid \delta\left(f, H_{n}\right)<k / 3\right\}
$$

(the symbol $\delta$ above is used for the metric on the function space $C(Y)$ induced by the sup norm) may be covered with finitely many closed balls with center in $\boldsymbol{H}_{n}$ and radius $(5 / 12) k$ (a trivial compactness argument). The inverse images (by $F$ ) of those balls would give a finite covering of $C_{n}$ with closed sets. If the interior of $C_{n}$ was nonempty then at least one of those closed sets would have an interior point. But then the local oscillation $\alpha_{F}$ would be at most $(10 / 12) k$ at such a point. This contradiction shows that $C_{n}$ has empty interior. Thus $\beta$ may choose his set to be disjoint from $C_{n}$ and we shall assume that he does so.

Let us choose $x_{\infty} \in \cap_{n=1}^{\infty} V_{n}=\cap_{n=1}^{\infty} U_{n}$ such that $x_{n}$ (the set of points chosen by $\alpha$ using his winning strategy $s$ ) accumulates to $x_{\infty}$. The sequence $F\left(x_{n}\right) \in C(Y)$ fulfills the conditions of the lemma and we may choose a subsequence $F\left(x_{n_{p}}\right)$ tending weakly to $F\left(x_{\infty}\right)$. But then suitable convex combinations of the $F\left(x_{n_{p}}\right)$ 's tend uniformly to $F\left(x_{\infty}\right)$. But the distance of $F\left(x_{\infty}\right)$ to any convex combination of the $F\left(x_{n}\right)$ 's is at least $k / 3$. This contradiction finishes the proof of Theorem 1.

3. Remarks on analogous results. While it seems likely that the conditions on $X$ can be relaxed (but probably not to only requiring $X$ to be a Baire space) the compactness assumption on $Y$ is very essential in the proof. If we use a different proof, however, we might prove a result for noncompact $Y$. Naturally the compactness condition must be replaced by some other strong condition; it seems, for example, unlikely that Theorem 1 holds for arbitrary complete metric spaces $X$ and $Y$. For a Hausdorff Baire space $X$ and a Hausdorff space $Y$ with some countability condition (weaker than second countability) $R$. E. Feiock has proved a result analogous to Theorem 1 (see [4]). The reader should consult also the paper by Calbrix and Troallic (see [1]).

We shall give an example due to J. Hoffmann-Jørgensen (oral communication) which shows that the main theorem is false even for compact range space provided this range space is "large". It seems quite probable that the condition on $Z$ to be metrizable cannot be relaxed very much, unless we put some "smallness" condition on $Z$.

$J$. Hoffmann-Jorgensen's example. We let $X=Y=[-1,1]$ and $Z$ be the space of mappings from $[-1,1]^{2}$ into $[-1,1]$ equipped with the pointwise topology. Thus 
$F(x, y)$ is a function of $(a, b) \in[-1,1]^{2}$ given by

$$
F(x, y)(a, b)=2(x-a)(y-b) /\left((x-a)^{2}+(y-b)^{2}\right)
$$

if this quotient is undefined we put it equal to zero. It is an easy exercise to verify that $F$ is separately continuous but not jointly continuous in any point.

4. Applications. We must confess that we know of no applications to automatic continuity theory where continuity is derived from purely algebraic properties of the mappings involved (and all topological assumptions placed on the spaces involved). There are several applications of the form that some continuity and some algebraic condition imply a strong form of continuity. As an example of such a theorem it is relatively easy to show (given Theorem 1 above) that if we have a locally compact topology on some group $G$ such that right and left translations are continuous, then the group is a locally compact group under the topology in question. This is due to Ellis (see [3]).

Such applications and also applications to show the existence of "many" dentable points for weakly compact subsets of not necessarily separable Fréchet spaces are discussed in detail in Namioka's paper (see [6]). We shall as an example show how the theorem can be used to prove very easily a deep result due to Glicksberg (see [5]).

THEOREM 2. Let $G$ be a locally compact abelian group and let $S \subseteq G$ be compact in the coarsest topology induced on $G$ by the dual group $\Gamma$ (the Bohr topology). Then $S$ is compact in $G$.

Proof. Let $H$ be a compact neighborhood in $\Gamma$. Let $A \subseteq H$ be a dense $G_{\delta}$ set such that $(\cdot, \cdot)$ is jointly continuous in $S \times A$ (restricted to $S \times H$ ). Some point $h \in A$ is an interior point of $H$ and for a suitable neighborhood $V$ of $h$ contained in $H$ we we have $|(s, v)-(s, h)|<\frac{1}{2}$ for all $v \in V$ and $s \in S$. But this shows that $S$ is contained in a compact set and therefore is compact since $S$ is trivially closed. This finishes the proof of Theorem 2.

ACKNOWLEDGements. The author is thankful to the referee for pointing out some errors in an earlier version of the paper and assisting with finding references. Moreover he is thankful to F. Topsøe and J. Hoffmann-Jørgensen who both encouraged this work.

AdDed IN Proof (March 1981). The attention of the author has been drawn (by F. Topsøe and J. Hoffmann-Jørgensen) to the surprising fact that for metrizable spaces the concept of $\alpha$-favorable in the Choquet sense implies $\tau$-well $\alpha$-favorable. To see this one simply chooses a winning strategy in the original Choquet sense, which has the property that for any consistent game, the diameter of the sets tends to zero. It is easy to see that for any choice of the points in the sets, we get a winning strategy for the $\tau$-game. As the real line with the Sorgenfrey topology shows this implication is not true for arbitrary (even completely regular) topological spaces. 


\section{REFERENCES}

1. J. Calbrix and J. P. Troallic, Applications séparément continues, C. R. Acad. Sci. Paris 288 (1979), 647-648.

2. Gustave Choquet, Lectures on analysis, Vol. 1, Benjamin, New York and Amsterdam, 1969.

3. R. Ellis, Locally compact transformation groups, Duke Math. J. 24 (1957), 119-125.

4. R. E. Feiock, Cluster sets and joint continuity, J. London Math. Soc. 7 (1973), 397-406.

5. I. Glicksberg, Uniform boundedness for groups, Canad. J. Math. 14 (1962), 269-276.

6. I. Namioka, Separate and joint continuity, Pacific J. Math. 51 (1974).

KбвENHAVNS UNIVERSITETS MATEMATISKe INSTITUT, UNIVERSITETSPARKEN 5, 2100 KбBENHAVN 6 , DENMARK 